\title{
Editorial for the Special Issue "Forestry Applications of Unmanned Aerial Vehicles (UAVs)"
}

\author{
Alessandro Matese \\ Institute of BioEconomy (IBE), National Research Council (CNR), Via Caproni 8, 50145 Florence, Italy; \\ alessandro.matese@cnr.it; Tel.: +39-055-3033711
}

Received: 2 April 2020; Accepted: 3 April 2020; Published: 5 April 2020

\begin{abstract}
Unmanned aerial vehicles (UAVs) are new platforms that have been increasingly used in the last few years for forestry applications that benefit from the added value of flexibility, low cost, reliability, autonomy, and capability of timely provision of high-resolution data. This special issue (SI) collects nine papers reporting research on different forestry applications using UAV imagery. The special issue covers seven Red-Green-Blue (RGB) sensor papers, three papers on multispectral imagery, and one further paper on hyperspectral data acquisition system. Several data processing and machine learning methods are presented. The special issue provides an overview regarding potential applications to provide forestry characteristics in a timely, cost-efficient way. With the fast development of sensors technology and image processing algorithms, the forestry potential applications will growing fast, but future work should consider the consistency and repeatability of these novel techniques.
\end{abstract}

Keywords: unmanned aerial vehicles (UAV); precision forestry; forestry applications; image processing; machine learning; RGB imagery

\section{Introduction}

Unmanned aerial vehicles (UAVs) are new platforms that have been increasingly used in the last few years for forestry applications that benefit from the added value of flexibility, low cost, reliability, autonomy, and capability of timely provision of high-resolution data. The main adopted image-based technologies are RGB, multispectral, and thermal infrared. LiDAR sensors are becoming commonly-used to improve the estimation of relevant plant traits. In comparison with other permanent ecosystems, forests are particularly affected by climatic changes due to the longevity of the trees, and the primary objective is the conservation and protection of forests. Nevertheless, forestry and agriculture both involve the cultivation of renewable raw materials - the difference is that forestry is less tied to economic aspects and this reflects the delay in using new monitoring technologies. The use of UAV in precision forestry has exponentially increased in the last years as demonstrated by a large number of papers published between 2018 and 2019, and more than 400 references are found searching for "UAV" + "forest" and considering articles, conference proceedings, and books.

The main forestry applications aim to inventory resources, map diseases, species classification, fire monitoring, and spatial gaps estimation. This Special Issue focused on new technologies (UAV and sensors) and innovative data elaboration methodologies (object recognition and machine vision) for forestry applications.

\section{Overview of Contributions}

VOSviewer software version 1.6.14 (Centre for Science and Technology Studies, Leiden University, The Netherlands) was used for a simple bibliometric map, shown in Figure 1. The software was developed for creating, visualizing, and exploring papers' bibliometrics. Term map offers overviews 
for identifying the structure of a topic and represents research topics where strongly related terms are located close to each other and the weaker the relationship is between terms, the greater the distance is between them. Terms were extracted in titles and abstracts of papers and represented in the map as circles.

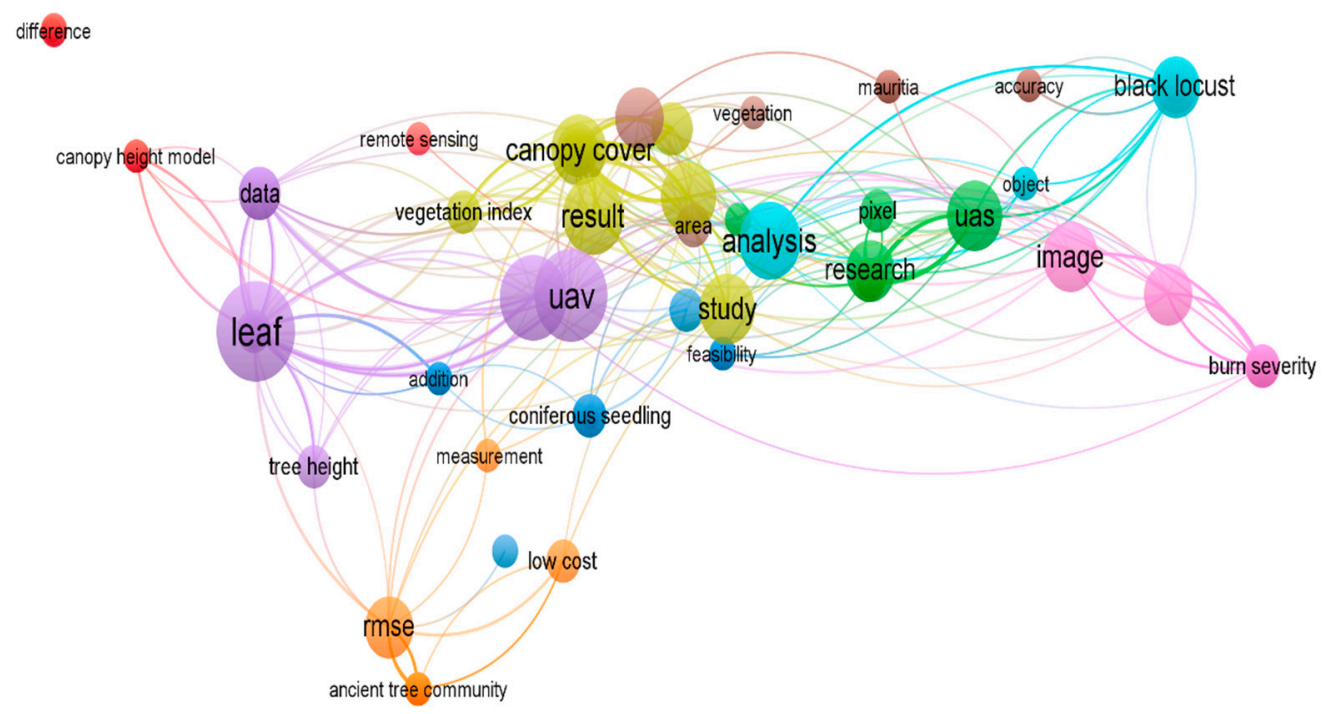

Figure 1. Term clustering map based on the SI publications. Different colors represent the terms belonging to different clusters. The size of the term is based on the number of occurrences. The connecting lines indicate the strongest co-occurrence links between terms.

Seven papers in this special issue reported results from UAV platform using RGB cameras [1-7], three paper used multispectral cameras [6,8,9], while one paper used a hyperspectral camera [6].

The aims of the papers were to test the feasibility of using UAVs to rapidly identify coniferous seedlings in replanted forest-harvest using an efficient sampling-based approach, consumer-grade cameras, and straightforward image handling, such as in [1]. The tree characteristics monitored were tree height, crown width, prediction of diameter at breast height (DBH), and tree age with low cost, high efficiency, and high precision in [2]. The development of a dataset called MauFlex related to Mauritia flexuosa palm, also known as "aguaje", was a study aimed at its conservation as it is a species poorly monitored because of the difficult access to these swamps. Moreover, a segmentation and measurement method for areas covered in Mauritia flexuosa palms using high-resolution aerial images acquired by UAVs was performed in this research [3]. One study evaluated the feasibility of adopting the low-cost, flexible, high-resolution, sensor-capable UAV platform for collecting reference data to use in thematic map accuracy assessments for complex environments [4]. Another research study focused on seed germination, stump shoot resprout, and spreading by root suckering of black locust in ten short rotation coppices [5]. The evaluation of density and canopy cover of western juniper in a treated (juniper removed) and an untreated watershed and an assesment of the effectiveness of using low altitude UAV-based imagery to measure juniper-sapling population density and canopy cover were also studied [6]. The investigation of UAV-based photogrammetric point clouds and hyperspectral imagery for characterizing seedling stands in leaf-off and leaf-on conditions were researched in [7]. The study of multispectral UAV images that can be used to classify burn severity, including the burned surface class, were considered in [8]. Lastly, the estimation of Chestnut pruning biomass through differences in the volume of canopy trees and an evaluation of the performance of an unsupervised segmentation methodology as a feasible tool for the analysis of large areas were considered in [9].

Various data processing methods were used in this Special Issue.

Image analysis workflow, where a three-step, object-based process consisting of image segmentation, automated classification using a classification and regression tree (CART) 
machine-learning algorithm, and the merging of adjacent image objects classified as 'seedlings' into single seedling objects, was conducted in [1]. The Structure-from- Motion/Multi-view Stereo (SfM-MVS) method was used to detect the feature points of the image, and the scale-invariant feature transform (SIFT) algorithm was used to detect feature points and generate feature vectors. Then, matching was carried out according to the feature vectors, and the RANSAC (random sample consensus) algorithm was used to delete the connection of the conflicting geometric features of corresponding feature points in [2]. Convolutional Neural Network $(\mathrm{CNN})$ based on the Deeplab v3+ architecture was completed. Images were acquired under different environment and light conditions using three different RGB cameras in [3]. Pixel-based and object-based classification reference data methods were used in [4]. Object-based image analysis (OBIA) and convolutional neural network (CNN) were used in [5]. Vegetation indices and support vector machine classification were used in [6]. Watershed segmentation method to delineate the tree canopy boundary at an individual tree level, and optimal bands for calculating vegetation indices were determined. Species classification using the random forest method was used in [7]. Maximum likelihood (MLH), spectral angle mapper (SAM), and thresholding of a normalized difference vegetation index (NDVI) were used as classifiers in [8]. Supervised and unsupervised crown segmentation with a double filtering process based on Canopy Height Model (CHM) and vegetation index threshold were used in [9].

Conflicts of Interest: The authors declare no conflict of interest.

\section{References}

1. Feduck, C.; McDermid, G.; Castilla, G. Detection of Coniferous Seedlings in UAV Imagery. Forests 2018, 9, 432. [CrossRef]

2. Qiu, Z.; Feng, Z.; Wang, M.; Li, Z.; Lu, C. Application of UAV Photogrammetric System for Monitoring Ancient Tree Communities in Beijing. Forests 2018, 9, 735. [CrossRef]

3. Morales, G.; Kemper, G.; Sevillano, G.; Arteaga, D.; Ortega, I.; Telles, J. Automatic Segmentation of Mauritia flexuosa in Unmanned Aerial Vehicle (UAV) Imagery Using Deep Learning. Forests 2018, 9, 736. [CrossRef]

4. Fraser, B.; Congalton, R. Evaluating the Effectiveness of Unmanned Aerial Systems (UAS) for Collecting Thematic Map Accuracy Assessment Reference Data in New England Forests. Forests 2019, 10, 24. [CrossRef]

5. Carl, C.; Lehmann, J.; Landgraf, D.; Pretzsch, H. Robinia pseudoacacia L. in Short Rotation Coppice: Seed and Stump Shoot Reproduction as well as UAS-based Spreading Analysis. Forests 2019, 10, 235. [CrossRef]

6. Durfee, N.; Ochoa, C.; Mata-Gonzalez, R. The Use of Low-Altitude UAV Imagery to Assess Western Juniper Density and Canopy Cover in Treated and Untreated Stands. Forests 2019, 10, 296. [CrossRef]

7. Imangholiloo, M.; Saarinen, N.; Markelin, L.; Rosnell, T.; Näsi, R.; Hakala, T.; Honkavaara, E.; Holopainen, M.; Hyyppä, J.; Vastaranta, M. Characterizing Seedling Stands Using Leaf-Off and Leaf-On Photogrammetric Point Clouds and Hyperspectral Imagery Acquired from Unmanned Aerial Vehicle. Forests 2019, $10,415$. [CrossRef]

8. Shin, J.; Seo, W.; Kim, T.; Park, J.; Woo, C. Using UAV Multispectral Images for Classification of Forest Burn Severity—A Case Study of the 2019 Gangneung Forest Fire. Forests 2019, 10, 1025. [CrossRef]

9. Di Gennaro, S.; Nati, C.; Dainelli, R.; Pastonchi, L.; Berton, A.; Toscano, P.; Matese, A. An Automatic UAV Based Segmentation Approach for Pruning Biomass Estimation in Irregularly Spaced Chestnut Orchards. Forests 2020, 11, 308. [CrossRef]

(C) 2020 by the author. Licensee MDPI, Basel, Switzerland. This article is an open access article distributed under the terms and conditions of the Creative Commons Attribution (CC BY) license (http://creativecommons.org/licenses/by/4.0/). 